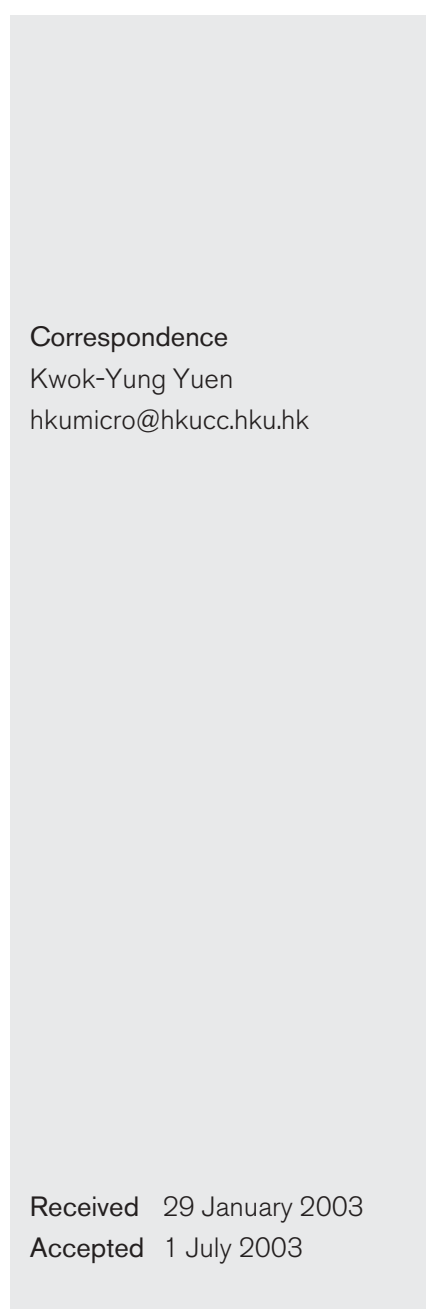

\title{
Geographical difference of disease association in Streptococcus bovis bacteraemia
}

\author{
Rodney A. Lee, ${ }^{1}$ Patrick C. Y. Woo, ${ }^{1}$ Amanda P. C. To, ${ }^{1}$ Susanna K. P. Lau, ${ }^{1}$ \\ Samson S. Y. Wong ${ }^{1}$ and Kwok-Yung Yuen ${ }^{1,2}$ \\ ${ }^{1}$ Department of Microbiology, The University of Hong Kong, University Pathology Building, \\ Queen Mary Hospital, Hong Kong \\ ${ }^{2}$ HKU-Pasteur Research Centre, Hong Kong
}

\begin{abstract}
From 1996 to 2001, 48 Streptococcus bovis strains were isolated from blood cultures of 37 patients in one hospital. Median patient age was 68 years (range: 1 day-88 years). The male : female ratio was 23 : 14. Most patients (97\%) had underlying diseases, including biliary tract disease in 14 (38\%), diabetes mellitus in 12 (32\%), liver parenchymal disease in seven (19\%), carcinoma of the colon in four (11\%) and other malignancies in four (11\%). No infective foci (indicative of primary bacteraemia) were identified in 15 patients (40\%) and 14 (38\%) had acute cholangitis/ cholecystitis, but only four (11\%) had infective endocarditis. Two (5\%), three (8\%) and 32 (87\%) patients had $S$. bovis of biotypes I, II/1 and II/2, respectively, and three (8\%), two (5\%) and 32 $(87 \%)$ patients had S. bovis of genotypes $1,2 \mathrm{a}$ and $2 \mathrm{~b}$, respectively. All isolates were sensitive to penicillin, cephalothin and vancomycin, 24 (65\%) were resistant to erythromycin and 15 (41\%) were resistant to clindamycin (these strains were also resistant to erythromycin). Thirteen isolates that were erythromycin- and clindamycin-resistant possessed the ermB gene, 10 possessed the ermT gene and one possessed both the ermB and ermT genes. Overall, seven patients (19\%) died. In contrast to most other reports from western countries, where carcinoma of the colon and infective endocarditis were the major underlying disease and infective focus associated with $S$. bovis bacteraemia, biliary tract disease and acute cholangitis and/or cholecystitis were the major underlying diseases associated with $S$. bovis bacteraemia in our locality.
\end{abstract}

\section{INTRODUCTION}

Streptococcus bovis is frequently recovered as a commensal in both humans and animals. Isolation of $S$. bovis from blood cultures of patients has a documented association with carcinoma of the colon and infective endocarditis (Klein et al., 1977, 1979; Steinberg \& Naggar, 1977; Murray \& Roberts, 1978; Reynolds et al., 1983; Kupferwasser et al., 1998). Two reports have also demonstrated an association of $S$. bovis bacteraemia with chronic liver parenchymal disease (Zarkin et al., 1990; Gonzalez-Quintela et al., 2001). Phenotypically, the API 20 STREP system (bioMérieux) subdivides $S$. bovis into three distinct biotypes, namely I, II/1 and II/2 (Ruoff et al., 1989). Many reports have identified S. bovis biotype I as the predominant isolate in cases of bacteraemia caused by the S. bovis group (Murray et al., 1999; Songy et al., 2002); it is also the biotype that is particularly associated with carcinoma of the colon and infective endocarditis (Murray et al., 1999). Genotypic studies that used DNA sequencing of a 500 bp fragment of the $16 \mathrm{~S}$ rRNA gene have shown that there is concordance between different biotypes and genotypes (Clarridge et al., 2001). Recently, based on a combination of DNA homology studies, whole-cell protein analysis and sequencing of the sodA gene, suggestions have been made for the revision of S. bovis taxonomy. Streptococcus gallolyticus, Streptococcus infantarius and Streptococcus pasteurianus have been proposed to replace $S$. bovis I, S. bovis II/1 and $S$. bovis II/2, respectively (Facklam, 2002).

One study showed a high incidence of erythromycin resistance in S. bovis (Teng et al., 2001). Despite in-depth phenotypic and genotypic characterization and disease association studies in western countries, none have attempted to study the epidemiology and disease association of $S$. bovis bacteraemia in countries outside North America and Europe.

In this study, we characterized $48 \mathrm{~S}$. bovis strains that were isolated from blood cultures of 37 bacteraemic patients over a 6-year period by using a combination of phenotypic and genotypic techniques. Epidemiology, underlying diseases, clinical disease associations and outcome of S. bovis bacteraemia in relation to the different biotypes and genotypes of $S$. bovis and molecular epidemiology of erythromycin resistance in $S$. bovis strains were investigated. 


\section{METHODS}

Patients and microbiological methods. The 37 patients in this study were hospitalized at the Queen Mary Hospital in Hong Kong during a 6year period (1996-2001). All clinical data were collected as described previously (Woo et al., 2001c). Suspect colonies were identified by standard conventional biochemical methods (Murray et al., 1999). In addition, the API 20 STREP system (bioMérieux) was used for identification of all Streptococcus-like (Streptococcus, Enterococcus, Granulicatella, Abiotrophia and Gemella) isolates and biotyping of S. bovis isolates. Antimicrobial susceptibility was tested by the KirbyBauer disc-diffusion method and results were interpreted according to NCCLS criteria (Bauer et al., 1966).

Extraction of bacterial DNA. Bacterial DNA extraction was modified from our previously published protocol (Woo et al., 1997). In brief, 80 $\mu \mathrm{l} \mathrm{NaOH}(0.05 \mathrm{M})$ was added to $20 \mu \mathrm{l}$ bacterial cells suspended in distilled water and the mixture was incubated at $60{ }^{\circ} \mathrm{C}$ for $45 \mathrm{~min}$, followed by addition of $6 \mu \mathrm{Tris} / \mathrm{HCl}(\mathrm{pH} 7 \cdot 0)$ to achieve a final $\mathrm{pH}$ of $8 \cdot 0$. The resultant mixture was diluted $100 \times$ and $5 \mu$ diluted extract was used for PCR.

Genotyping by 165 rRNA gene sequencing. PCR amplification and DNA sequencing of a $500 \mathrm{bp}$ fragment of the $16 \mathrm{~S}$ rRNA gene were performed as described previously (Woo et al., 2000, 2001a, b, 2002a, b, c; Yuen et al., 2001; Lau et al., 2002) by using primers LPW601 (5'ATGGGAGAGTTTGATCCCTG-3') and LPW602 (5'-TACCCG CGGCTGCTGGCACG-3') (Gibco-BRL). Sequences of PCR products were compared with known $16 \mathrm{~S}$ rRNA gene sequences in GenBank by multiple sequence alignment with the CLUSTAL $\mathrm{W}$ program (Thompson et al., 1994) and phylogenetic tree construction was performed by using PileUp with GrowTree (Genetics Computer Group).

erm gene sequencing. PCR amplification and DNA sequencing of the erm genes were performed according to a previously published method (Teng et al., 2001) by using primers LPW632 (5'-GAAAAGGTACT CAACCAAATA-3') and LPW633 (5'-AGTAACGGTACTTAAATT GTTTAC-3') for the ermB gene and LPW636 (5'-GCTAATATTGTT TAAATCGTCAATTCC-3') and LPW637 (5'-GGATCAGGAAAAG GACATTTTAC-3') for the erm $T$ gene (Gibco-BRL). Sequences of PCR products were compared with known erm gene sequences in GenBank by multiple sequence alignment with the CLUSTAL W program (Thompson et al., 1994) and phylogenetic tree construction was performed by using PileUp with GrowTree (Genetics Computer Group)

\section{RESULTS AND DISCUSSION}

In a 6-year period (1996-2001), 48 strains of S. bovis were isolated from blood cultures of 37 patients and were identified as $S$. bovis by the API 20 STREP system. S. bovis strains recovered from the same patient exhibited the same biotype and genotype based on 16S rRNA gene sequence analysis. Characteristics of the 37 patients with $S$. bovis bacteraemia were as follows: median age was 68 years (range: 1 day- 88 years) and 23 patients $(63 \%)$ were over 60 years of age. The male : female ratio was $23: 14$. Most patients (97\%) had underlying diseases: the major underlying diseases included biliary tract disease in $14(38 \%)$, diabetes mellitus in $12(32 \%)$, liver parenchymal disease in seven $(19 \%)$, carcinoma of the colon in four $(11 \%)$ and other malignancies in four $(11 \%)$. The only patient without underlying disease was a 2-year-old boy with gastroenteritis; the $S$. bovis strain in this patient's blood had probably translocated from the gastrointestinal tract as a result of intestinal inflammation. No infective foci (indicative of primary bacteraemia) were identified in 15 patients ( $40 \%)$, whereas $14(38 \%)$ had acute cholangitis/cholecystitis, four (11\%) had infective endocarditis, one $(3 \%)$ had secondary peritonitis due to a perforated appendix, one ( $3 \%$ ) had neonatal meningitis, one (3\%) had spontaneous bacterial peritonitis and one (3\%) had cellulitis. All patients had community-acquired S. bovis bacteraemia. S. bovis was recovered from a single blood culture from 32 patients $(87 \%)$, whereas the organism was isolated from multiple blood cultures from five patients $(13 \%)$. Two (5\%), three (8\%) and $32(87 \%)$ patients had $S$. bovis of biotypes I, II/ 1 and II/2, respectively. Three ( $8 \%)$, two $(5 \%)$ and $32(87 \%)$ patients had S. bovis of genotypes 1 , $2 \mathrm{a}$ and $2 \mathrm{~b}$, respectively (Fig. 1). S. bovis was the only bacterium recovered from blood cultures of 25 patients

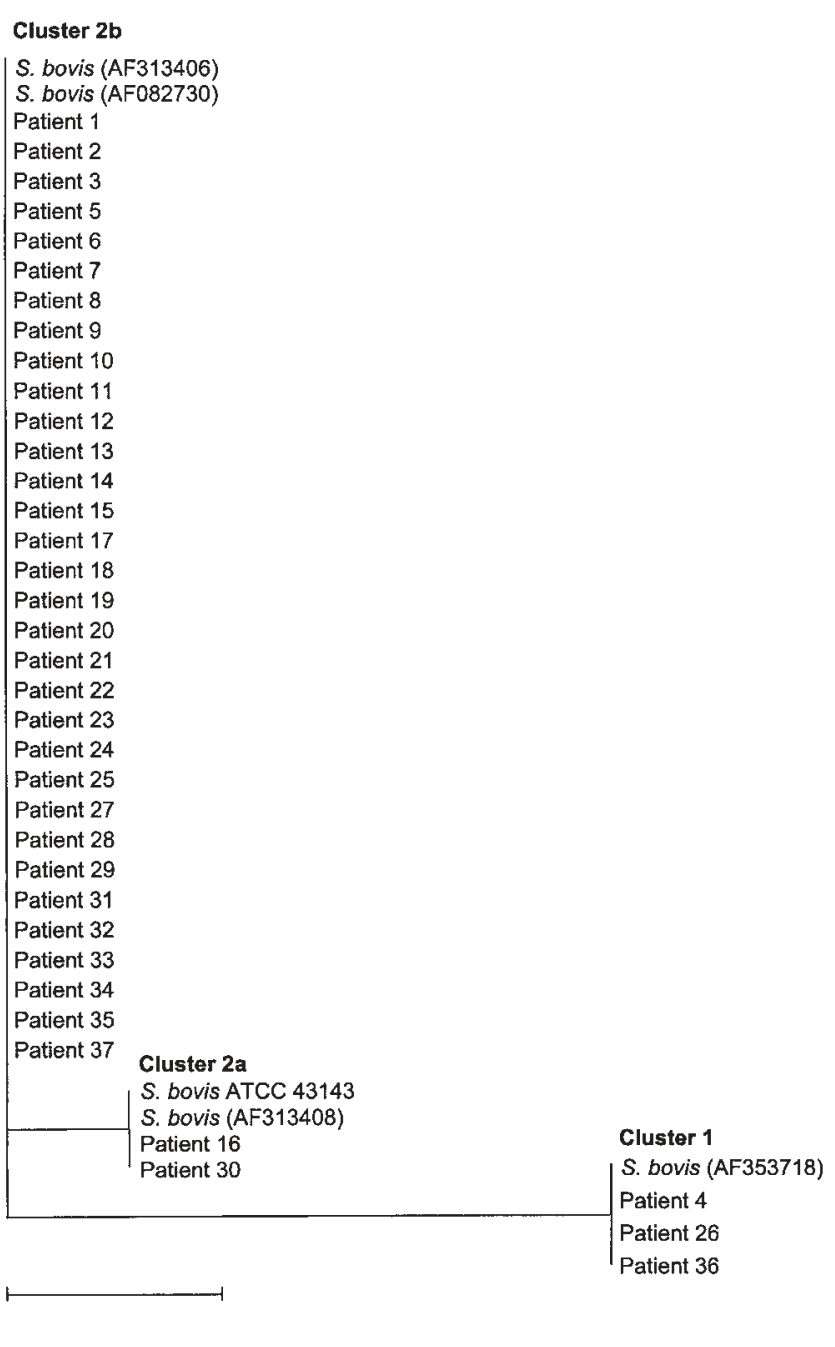

Fig. 1. Phylogenetic tree showing the relationship of $S$. bovis strains recovered from 37 patients. The tree was inferred from $500 \mathrm{bp}$ of the $16 S$ rRNA gene sequence by the neighbour-joining method. Names and accession numbers are given as cited in GenBank. Bar, 1 substitution per 100 bases (estimated by using the Jukes-Cantor correction). 
(68\%) whereas in 12 patients (32\%), other bacteria were recovered concomitantly with $S$. bovis from blood cultures (Escherichia coli in five patients, Citrobacter farmeri in one patient, Klebsiella pneumoniae in one patient, Escherichia coli and K. pneumoniae in two patients, Escherichia coli and Enterococcus faecalis in one patient, Escherichia coli, Bacteroides fragilis and Fusobacterium sp. in one patient and Escherichia coli, K. pneumoniae and Aeromonas hydrophila in one patient). Only one patient, with neonatal meningitis, had $S$. bovis recovered at other sites in addition to blood (cerebrospinal fluid and nasal swab). Isolates recovered from all patients were sensitive to penicillin, cephalothin and vancomycin, whereas those recovered from 24 patients (65\%) were resistant to erythromycin and those recovered from 15 patients $(41 \%)$ were resistant to clindamycin (these strains were also resistant to erythromycin). Overall, seven patients (19\%) died.

The erm genes of the 24 S. bovis isolates that were resistant to erythromycin were amplified and sequenced. Thirteen iso- lates that were erythromycin- and clindamycin-resistant possessed the ermB gene, 10 possessed the erm $T$ gene and one possessed both the ermB and ermT genes (Fig. 2).

In this study, we describe the characterization of $48 \mathrm{~S}$. bovis strains isolated from 37 patients with S. bovis bacteraemia. Similarly to a recent study that described the characterization of 22 clinical strains of S. bovis (Clarridge et al., 2001), we also demonstrated concordance between the different biotypes (as identified by the API 20 STREP system) and genotypes (based on 16S rRNA gene sequencing). Similarly to this recent study and another in Taiwan (in which $88 \%$ of 60 blood culture isolates were of biotype II/2) (Teng et al., 2001), biotype II/ 2 or genotype 2 b was the most predominant type of $S$. bovis found in our patients (Table 1). This is different from the findings of previous studies that demonstrated that biotype I was the predominant type of $S$. bovis isolated from their bacteraemic patients (Ruoff et al., 1989).

In contrast to previous studies that described a strong association between $S$. bovis bacteraemia and carcinoma of

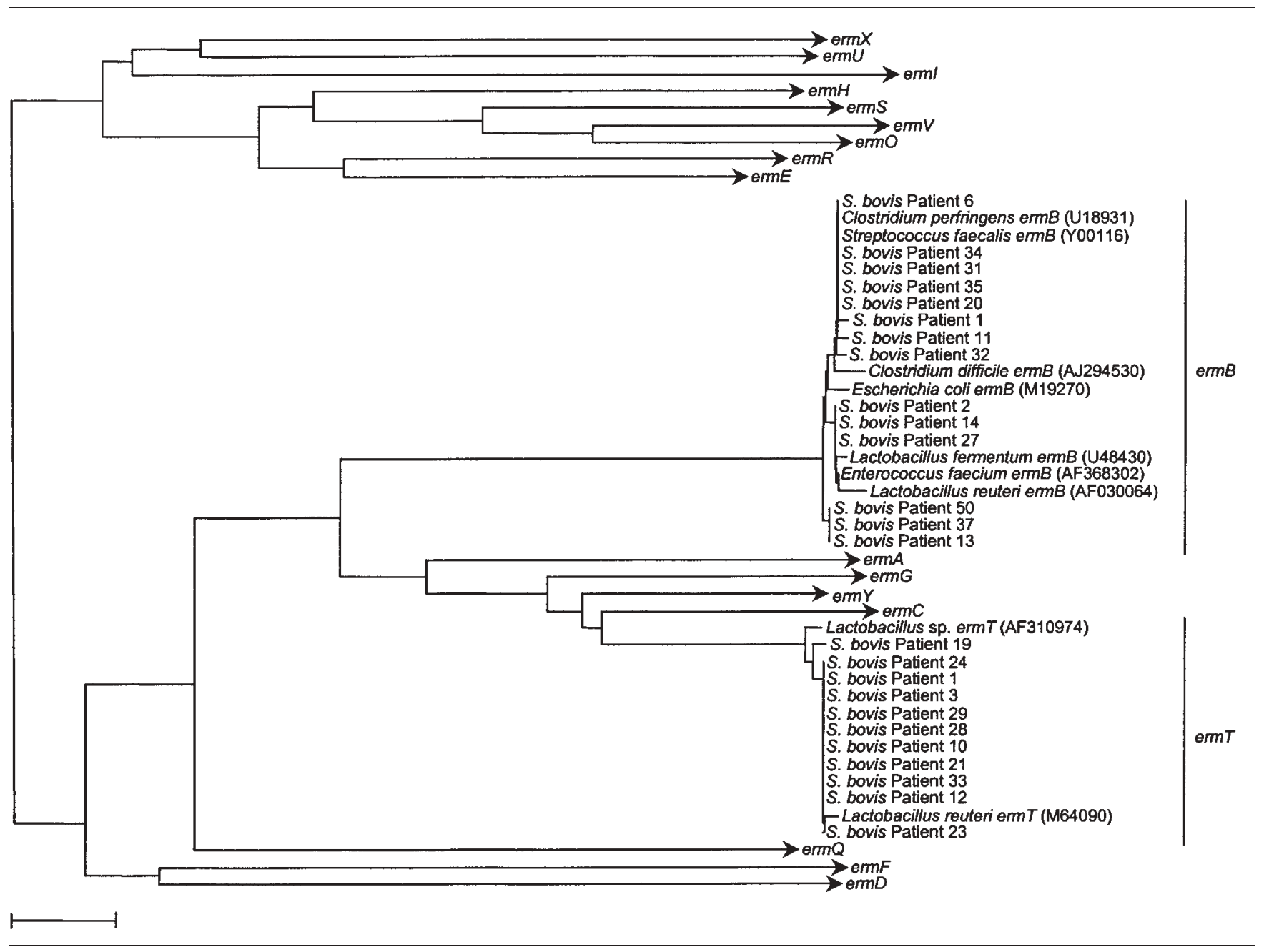

Fig. 2. Phylogenetic tree showing the relationship of the amino acid sequences of the ermB and ermT genes of the 24 erythromycinresistant S. bovis strains to those of other erm genes. The tree was inferred from 174 aa sequence data by the neighbour-joining method. Names and accession numbers are given as cited in GenBank. Bar, 5 substitutions per 100 amino acids (estimated by using the Kimura correction). 
Table 1. Comparison of characteristics from major studies of Streptococcus bovis bacteraemia

References: 1, Murray \& Roberts, 1978; 2, Reynolds et al., 1983; 3, Pigrau et al., 1988; 4, Ruoff et al., 1989; 5, Zarkin et al., 1990; 6, Clarridge et al., 2001; 7, Gonzalez-Quintela et al., 2001; 8, Teng et al., 2001; 9, present study. Unless stated, data are number of patients with the characteristic, with percentage in parentheses. NM, Not mentioned.

\begin{tabular}{|c|c|c|c|c|c|c|c|c|c|}
\hline \multirow[t]{2}{*}{ Characteristic } & \multicolumn{9}{|c|}{ Major studies on S. bovis bacteraemia } \\
\hline & 1 & 2 & 3 & 4 & 5 & 6 & 7 & 8 & 9 \\
\hline No. patients & 36 & 19 & 16 & 38 & 92 & 12 & 20 & 60 & 37 \\
\hline Mean age (years) & 61 & NM & 49 & 67 & 57 & NM & 62 & NM & 61 \\
\hline \multicolumn{10}{|l|}{ Sex: } \\
\hline Male & $19(53)$ & $11(58)$ & $8(50)$ & $21(55)$ & $45(49)$ & NM & $13(65)$ & NM & $23(62)$ \\
\hline Female & $17(47)$ & $8(42)$ & $8(50)$ & $17(45)$ & $47(51)$ & NM & $7(35)$ & NM & $14(38)$ \\
\hline \multicolumn{10}{|l|}{ Underlying diseases: } \\
\hline Liver parenchymal disease & $0^{b \star}(0)$ & $0^{a}(0)$ & $4(25)$ & NM & $45^{c}(47)$ & $0(0)$ & $11^{b}(55)$ & NM & $7(19)$ \\
\hline Biliary tract disease & $2^{d}(6)$ & $0^{d}(0)$ & $3(19)$ & NM & $3^{d}(3)$ & $0^{b}(0)$ & $0^{d}(0)$ & NM & $14(38)$ \\
\hline Colonic carcinoma & $5(14)$ & $2(11)$ & $1(6)$ & $15^{c}(40)$ & $16(17)$ & $1(8)$ & $6(30)$ & NM & $4(11)$ \\
\hline Benign lower gastrointestinal tract diseases & $10^{c}(28)$ & $11^{d}(58)$ & NM & NM & $6(7)$ & NM & $0(0)$ & NM & $1(3)$ \\
\hline \multicolumn{10}{|l|}{ Diagnosis: } \\
\hline Primary bacteraemia & $7^{a}(19)$ & $2^{a}(11)$ & $3(19)$ & NM & $56^{a}(61)$ & $6(50)$ & NM & NM & $15(41)$ \\
\hline Cholangitis/cholecystitis & $2^{d}(6)$ & $0^{d}(0)$ & $3(19)$ & NM & $3^{d}(3)$ & $0^{b}(0)$ & $0^{d}(0)$ & NM & $14(38)$ \\
\hline Infective endocarditis & $26^{d}(72)$ & $17^{d}(47)$ & $5(31)$ & $19^{d}(50)$ & $26^{a}(28)$ & $4(33)$ & $10^{d}(50)$ & NM & $4(11)$ \\
\hline \multicolumn{10}{|l|}{ Biotypes of $S$. bovis: } \\
\hline I & NM & NM & NM & $17^{d}(45)$ & NM & $1(8)$ & NM & $4(7)$ & $2(5)$ \\
\hline II/1 & NM & NM & NM & $12^{a}(32)$ & NM & $1(8)$ & NM & $3(5)$ & $3(8)$ \\
\hline $\mathrm{II} / 2$ & NM & NM & NM & $9^{d}(24)$ & NM & $10(83)$ & NM & $53(88)$ & $32(87)$ \\
\hline Mortality & $3(8)$ & NM & $5(31)$ & $9(24)$ & NM & NM & $8(40)$ & NM & $7(19)$ \\
\hline
\end{tabular}

${ }^{\star}$ Statistically significant when compared to the present study by using a $\chi^{2}$ test at the following levels: $a, P<0 \cdot 05 ; b, P<0 \cdot 01 ; c, P<0 \cdot 005 ; d, P<0 \cdot 001$.

the colon and infective endocarditis (Steinberg \& Naggar, 1977; Klein et al., 1977, 1979; Murray \& Roberts, 1978; Reynolds et al., 1983; Kupferwasser et al., 1998), the association between S. bovis bacteraemia and these clinical conditions was not strong in our population (Table 1). Previous studies have shown that associations between $S$. bovis bacteraemia and carcinoma of the colon and infective endocarditis were even higher for S. bovis biotype I isolates, with $94 \%$ association of S. bovis biotype I bacteraemia with infective endocarditis and $71 \%$ association of $S$. bovis biotype I bacteraemia with colonic carcinoma (Murray et al., 1999). On the other hand, for S. bovis isolates of biotype II, association between bacteraemia and infective endocarditis was only $18 \%$ and that between bacteraemia and carcinoma of the colon was only $17 \%$ (Murray et al., 1999). As our S. bovis strains mainly belonged to biotype II/2, it is not surprising that we have found a weak association between $S$. bovis bacteraemia and infective endocarditis and carcinoma of the colon. In the present study, the rate of endocarditis and carcinoma of the colon was only $11 \%$. Interestingly, none of the four patients with infective endocarditis and the four with carcinoma of the colon had bacteraemia caused by $S$. bovis biotype I.

Biliary tract disease and acute cholangitis and/or cholecystitis were the predominant underlying diseases and diagnoses associated with $S$. bovis bacteraemia in our locality. An association between $S$. bovis bacteraemia and chronic liver parenchymal disease has been reported previously (Table 1) (Zarkin et al., 1990; Gonzalez-Quintela et al., 2001). Pigrau et al. (1988) first noted that four of 16 cases of S. bovis bacteraemia had liver cirrhosis; subsequently, in a study by Zarkin et al. (1990), evidence of liver parenchymal disease was observed in 45 of 92 patients with S. bovis bacteraemia. More recently, Gonzalez-Quintela et al. (2001) described 11 of 20 patients with $S$. bovis bacteraemia that had chronic liver parenchymal disease. In the present study, 14 of 37 patients $(38 \%)$ had biliary tract disease and the same number of patients had acute cholangitis and/or cholecystitis. We speculate that the association of biliary tract disease with $S$. bovis bacteraemia is due to the following facts: firstly, that biliary tract disease is prevalent in our locality. In one study, it was demonstrated that biliary tract sepsis is the second most common cause of community-acquired bacteraemia after urinary tract infection (French et al., 1990). This uniquely high incidence of biliary sepsis in this part of the world is attributed to high prevalence of clonorchiasis and recurrent pyogenic cholangitis (Lo et al., 1997; Woo et al., 1998). Secondly, in contrast to most $\alpha$-haemolytic streptococci, $S$. bovis is able to grow in bile (Luk et al., 1998). This property 
renders it able to survive in bile and cause acute cholecystitis and cholangitis, especially in the presence of pre-existing biliary tract disease. Therefore, in our locality, biliary tract disease should be actively looked for in any future case of unexplained $S$. bovis bacteraemia.

The ermB and erm $T$ genes in the S. bovis isolates studied are highly homologous to those found in other bacteria of the gastrointestinal tract. Incidence and molecular epidemiology of erythromycin resistance in the present study are similar to those reported in a recent study from Taiwan (Teng et al., 2001). Similarly to the Taiwan study, which reported that $63 \%$ of S. bovis bacteraemic isolates were erythromycinresistant, $65 \%$ of $S$. bovis isolates in our locality were erythromycin-resistant. Furthermore, 58 and $46 \%$ of our erythromycin-resistant isolates contained the ermB or erm $T$ genes, respectively, which is also similar to the corresponding incidences of 63 and $37 \%$ reported in that study. The ermB and ermT genes in S. bovis showed high homology to the ermB genes in Clostridium difficile, Clostridium perfringens, Lactobacillus reuteri, Streptococcus agalactiae, Enterococcus faecalis, Enterococcus faecium, Lactobacillus fermentum and Escherichia coli and the ermT genes in L. reuteri and another Lactobacillus species, which are all bacteria from the gastrointestinal tract (Fig. 2). We therefore speculate that these genes in S. bovis were acquired through horizontal gene transfer from other bacteria that reside in the gastrointestinal tract.

\section{ACKNOWLEDGEMENTS}

This work is partly supported by the University Development Fund, University Research Grant Council and the Committee for Research and Conference Grant, The University of Hong Kong.

\section{REFERENCES}

Bauer, A. W., Kirby, W. M. M., Sherris, J. C. \& Turck, M. (1966). Antibiotic susceptibility testing by a standardized single disk method. Am J Clin Pathol 45, 493-496.

Clarridge, J. E., 3rd, Attorri, S. M., Zhang, Q. \& Bartell, J. (2001). 16S ribosomal DNA sequence analysis distinguishes biotypes of Streptococcus bovis: Streptococcus bovis biotype II/2 is a separate genospecies and the predominant clinical isolate in adult males. J Clin Microbiol 39, $1549-1552$.

Facklam, R. (2002). What happened to the streptococci: overview of taxonomic and nomenclature changes. Clin Microbiol Rev 15, 613-630.

French, G. L., Cheng, A. F., Duthie, R. \& Cockram, C. S. (1990). Septicaemia in Hong Kong. J Antimicrob Chemother 25 (Suppl. C), 115-125.

Gonzalez-Quintela, A., Martinez-Rey, C., Castroagudin, J. F., RajoIglesias, M. C. \& Dominguez-Santalla, M. J. (2001). Prevalence of liver disease in patients with Streptococcus bovis bacteraemia. J Infect 42, $116-119$

Klein, R. S., Recco, R. A., Catalano, M. T., Edberg, S. C., Casey, J. I. \& Steigbigel, N. H. (1977). Association of Streptococcus bovis with carcinoma of the colon. N Engl J Med 297, 800-802.

Klein, R. S., Catalano, M. T., Edberg, S. C., Casey, J. I. \& Steigbigel, N. H. (1979). Streptococcus bovis septicemia and carcinoma of the colon. Ann Intern Med 91, 560-562.
Kupferwasser, I., Darius, H., Muller, A. M., Mohr-Kahaly, S., Westermeier, T., Oelert, H., Erbel, R. \& Meyer, J. (1998). Clinical and morphological characteristics in Streptococcus bovis endocarditis: a comparison with other causative microorganisms in 177 cases. Heart 80, 276-280.

Lau, S. K. P., Woo, P. C. Y., Woo, G. K. S. \& Yuen, K.-Y. (2002). Catheterrelated Microbacterium bacteremia identified by $16 \mathrm{~S}$ rRNA gene sequencing. J Clin Microbiol 40, 2681-2685.

Lo, C. M., Fan, S. T. \& Wong, J. (1997). The changing epidemiology of recurrent pyogenic cholangitis. Hong Kong Med J 3, 302-304.

Luk, W. K., Liu, C. L., Yuen, K.-Y., Wong, S. S. Y., Woo, P. C. Y. \& Fan, S. T. (1998). Biliary tract infection due to bile-soluble bacteria: an intriguing paradox. Clin Infect Dis 26, 1010-1012.

Murray, H. W. \& Roberts, R. B. (1978). Streptococcus bovis bacteremia and underlying gastrointestinal disease. Arch Intern Med 138, 1097-1099.

Murray, P. R., Baron, E. J., Pfaller, M. A., Tenover, F. C. \& Yolken, R. H. (1999). Manual of Clinical Microbiology, 7th edn. Washington, DC: American Society for Microbiology.

Pigrau, C., Lorente, A., Pahissa, A. \& Martinez-Vazquez, J. M. (1988). Streptococcus bovis bacteremia and digestive system neoplasms. Scand J Infect Dis 20, 459-460.

Reynolds, J. G., Silva, E. \& McCormack, W. M. (1983). Association of Streptococcus bovis bacteremia with bowel disease. J Clin Microbiol 17, 696-697.

Ruoff, K. L., Miller, S. I., Garner, C. V., Ferraro, M. J. \& Calderwood, S. B. (1989). Bacteremia with Streptococcus bovis and Streptococcus salivarius: clinical correlates of more accurate identification of isolates. J Clin Microbiol 27, 305-308.

Songy, W. B., Ruoff, K. L., Facklam, R. R., Ferraro, M. J. \& Falkow, S. (2002). Identification of Streptococcus bovis biotype I strains among $S$. bovis clinical isolates by PCR. J Clin Microbiol 40, 2913-2918.

Steinberg, D. \& Naggar, C. Z. (1977). Streptococcus bovis endocarditis with carcinoma of the colon. N Engl J Med 297, 1354-1355.

Teng, L.-J., Hsueh, P.-R., Ho, S.-W. \& Luh, K.-T. (2001). High prevalence of inducible erythromycin resistance among Streptococcus bovis isolates in Taiwan. Antimicrob Agents Chemother 45, 3362-3365.

Thompson, J. D., Higgins, D. G. \& Gibson, T. J. (1994). CLUSTAL W: improving the sensitivity of progressive multiple sequence alignment through sequence weighting, position-specific gap penalties and weight matrix choice. Nucleic Acids Res 22, 4673-4680.

Woo, P. C. Y., Lo, C. Y., Lo, S. K. \& 7 other authors (1997). Distinct genotypic distributions of cytomegalovirus (CMV) envelope glycoprotein in bone marrow and renal transplant recipients with CMV disease. Clin Diagn Lab Immunol 4, 515-518.

Woo, P. C. Y., Lie, A. K. W., Yuen, K.-Y., Wong, S. S. Y., Lee, C. K. \& Liang, R. H. S. (1998). Clonorchiasis in bone marrow transplant recipients. Clin Infect Dis 27, 382-384.

Woo, P. C. Y., Tsoi, H.-W., Leung, K.-W., Lum, P. N. L., Leung, A. S. P., Ma, C.-H., Kam, K.-M. \& Yuen, K.-Y. (2000). Identification of Mycobacterium neoaurum isolated from a neutropenic patient with catheter-related bacteremia by 16 S rRNA sequencing. J Clin Microbiol 38, 3515-3517.

Woo, P. C. Y., Fung, A. M. Y., Lau, S. K. P., Wong, S. S. Y. \& Yuen, K.-Y. (2001a). Group G beta-hemolytic streptococcal bacteremia characterized by $16 \mathrm{~S}$ ribosomal RNA gene sequencing. J Clin Microbiol 39, 3147-3155.

Woo, P. C. Y., Fung, A. M. Y., Wong, S. S. Y., Tsoi, H.-W. \& Yuen, K.-Y. (2001b). Isolation and characterization of a Salmonella enterica serotype Typhi variant and its clinical and public health implications. J Clin Microbiol 39, 1190-1194. 
Woo, P. C. Y., Wong, S. S. Y., Lum, P. N. L., Hui, W.-T. \& Yuen, K.-Y. (2001c). Cell-wall-deficient bacteria and culture-negative febrile episodes in bone-marrow-transplant recipients. Lancet 357, 675-679.

Woo, P. C. Y., Leung, K.-W., Wong, S. S. Y., Chong, K. T. K., Cheung, E. Y. L. \& Yuen, K.-Y. (2002a). Relatively alcohol-resistant mycobacteria are emerging pathogens in patients receiving acupuncture treatment. J Clin Microbiol 40, 1219-1224.

Woo, P. C. Y., Tam, D. M. W., Leung, K.-W., Lau, S. K. P., Teng, J. L. L., Wong, M. K. M. \& Yuen, K.-Y. (2002b). Streptococcus sinensis sp. nov., a novel species isolated from a patient with infective endocarditis. J Clin Microbiol 40, 805-810.
Woo, P. C. Y., Fung, A. M. Y., Lau, S. K. P. \& Yuen, K.-Y. (2002c). Identification by $16 \mathrm{~S}$ rRNA gene sequencing of Lactobacillus salivarius bacteremic cholecystitis. J Clin Microbiol 40, 265-267.

Yuen, K.-Y., Woo, P. C. Y., Teng, J. L. L., Leung, K.-W., Wong, M. K. M. \& Lau, S. K. P. (2001). Laribacter hongkongensis gen. nov., sp. nov., a novel gram-negative bacterium isolated from a cirrhotic patient with bacteremia and empyema. J Clin Microbiol 39, 4227-4232.

Zarkin, B. A., Lillemoe, K. D., Cameron, J. L., Effron, P. N., Magnuson, T. H. \& Pitt, H. A. (1990). The triad of Streptococcus bovis bacteremia, colonic pathology, and liver disease. Ann Surg 211, 786-791. 